\title{
Validación de una versión en español de la Escala de Conflicto Decisional
}

\author{
Mila U rrutia ${ }^{1 a}$, Solange Campos ${ }^{1 b}$, Annette $0^{\prime}$ Connor $^{2 c}$. \\ Validation of a Spanish version \\ of the Decisional Conflict Scale
}

Background: In Chile, in approximately $50 \%$ of nursing students, nursing was not their first choice as career. Usually, during the first year, these students must decide whether they would like to continue in the same career. A valid tool is needed to identify decisional conflicts and their contributing factors among these students and to develop an appropriate strategy to support them during their decision-making process. Aim: To translate into Spanish and validate the Generic Decisional Conflict Scale (DCS). Material and methods: The DCS was translated from English to Spanish and was used with 331 first-year nursing students at the Pontificia Universidad Católica de Chile. The scale was assessed for validity and reliability using statistical tests, including factor analysis and Cronbach alpha test. Results: The Spanish version of the DCS had acceptable validity and reliability. Factorial analysis identified four factors and only the item: "advice" loaded the other factors. Cronbach alpha was 0.80 . Conclusions: DCS is a valid and useful instrument to identify decisional conflicts and contributing factors to continue studies among nursing students (Rev Méd Chile 2008; 136:1439-47).

(Key words: Decision makings; Education, nursing; student dropouts)

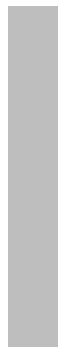

Recibido el 16 de agosto, 2007. Aceptado el 5 de agosto, 2008.

Financiamiento: Proyecto "Making choices, making decisions: a client-provider partnership in Canadian/Chilean technology transfer (DECIDE)".

${ }^{1}$ Escuela de Enfermería, Pontificia Universidad Católica de Chile. Santiago de Chile. ${ }^{2}$ Escuela de Enfermería, Facultad de Ciencias de la Salud, Departamento de Epidemiología y Salud Comunitaria, Facultad de Medicina, Universidad de Ottawa. Canadá.

aEnfermera, Magíster en Salud de la Comunidad

bEnfermera-matrona, Magíster en Psicología Social, Escuela de Enfermería, Pontificia Universidad Católica de Chile

${ }^{\mathrm{C}} \mathrm{RN}$. BScN, MScN, PhD.

$\mathrm{E}_{\mathrm{s}}^{\mathrm{n}}$ Chile, algunos de los estudiantes que ingresan a la carrera de enfermería, lo hacen sólo por su deseo de ingresar a la Universidad, y no están seguros de su elección respecto a la carrera.

Correspondencia a: Mila Urrutia. Vicuña Mackenna 4860. Santiago. Fono: 3547969. Fax: 3547025.

E mail: murrutia@uc.cl
El sistema tradicional de selección pondera las notas de enseñanza media, el puntaje obtenido en la prueba nacional de selección universitaria y la prioridad definida por el postulante. Los alumnos estiman su puntaje y postulan considerando los puntajes históricos de ingreso a las diferentes carreras y no necesariamente su verdadera preferencia. A la carrera de Enfermería de la Pontificia Universidad Católica de Chile ingresa un prome- 
dio de 100 alumnos. Aproximadamente para 50\% de ellos, enfermería no es su primera prioridad ${ }^{1-3}$. Por ello, interesa conocer la proporción de alumnos que ha ingresado a la carrera y que presenta conflicto decisional respecto a la decisión de continuar en ella y los factores que están contribuyendo a este conflicto, con el fin de diseñar estrategias orientadas a apoyarlos en este proceso de toma de decisión. Sin embargo, actualmente no se cuenta con un instrumento validado que permita identificar a dichos alumnos.

Janis y Mann definen conflicto decisional como la presencia de tendencias opuestas al interior del individuo que lo llevan a aceptar 0 rechazar un curso de acción determinada ${ }^{4}$. Para $\mathrm{O}^{\prime}$ Connor ${ }^{5}$, el conflicto decisional es un estado de incertidumbre acerca de cuál curso de acción tomar debido a que las diferentes alternativas ofrecen beneficios y riesgos.

La Escala de Conflicto Decisional (ECD) fue adaptada de la Decisional Confict Scale (DCS $)^{5}$, instrumento que se basa en el Modelo Conceptual de Apoyo a la Toma de Decisiones en Salud de Ottawa ${ }^{6}$ y su propósito es identificar los conflictos decisionales y los factores que los influyen. La DCS fue desarrollada por O'Connor en respuesta a la falta de instrumentos disponibles para evaluar la necesidad de apoyo decisional de los usuarios en salud ${ }^{5}$. Sin embargo, el marco de referencia utilizado para su construcción es amplio ${ }^{6}$ y permite su aplicación a diferentes contextos.

La DCS fue validada por su autora en 1995 y demostró estabilidad. El coeficiente de correlación entre test y retest fue no significativo $(0,81)$, la consistencia interna fue alta para la escala total (alpha entre 0,78 y 0,92) (5) y su capacidad de discriminación fue significativa $(p<0,0002)^{5}$.

A nivel internacional, la DCS fue utilizada en estudios sobre vacunación contra la influenza y prueba de tamizaje de cáncer de mama ${ }^{5}$. El primero se realizó en tres grupos: estudiantes de ciencias de la salud ( $\mathrm{n}=151$ ), funcionarios de servicios de salud $(n=115)$ y pacientes con problemas cardíacos y respiratorios ( $\mathrm{n}=283)$, en Ottawa, Ontario, Canadá. El segundo se realizó con mujeres entre 50 y 60 años de edad en Ottawa. La escala fue validada en dos muestras de pacientes oncológicos alemanes. Se obtuvo un coeficiente de confiabilidad de 0,52 y 0,84 y la validez de constructo fue parcialmente confirma- $\mathrm{da}^{7}$. Ha sido utilizada en diversos estudios con todos sus ítems ${ }^{8-10}$ o con algunos de sus ítems ${ }^{11}$. También se han utilizado las subescalas independientemente ${ }^{12-14}$.

El Modelo Conceptual de Apoyo a la Toma de Decisiones en Salud de Ottawa ha sido utilizado en población chilena a través del proyecto Elecciones y Decisiones en Salud: una alianza entre profesionales y usuarios (DECIDE) desarrollado en la Escuela de Enfermería de la Pontificia Universidad Católica de Chile con el apoyo de la Universidad de Ottawa y financiado por la Agencia Canadiense de Desarrollo Internacional (ACDI) entre los años 1999 y 2004. El proyecto trabajó con 6 escuelas de enfermería del país (universidades de Antofagasta, La Serena, Los Andes, Bío-Bío, Concepción y Austral) y en la mayoría de los estudios realizados se aplicó el cuestionario Valoración de Necesidades de Apoyo Decisional ${ }^{15-17}$.

El propósito del presente trabajo fue la traducción, adaptación y validación de la DCS, para contar con un instrumento que permitiera identificar conflicto decisional y factores que lo influyen en la decisión de mantenerse en la carrera de enfermería de los alumnos que ingresan a ella.

\section{MATERIAL Y MÉTODO}

Descripción de la ECD. La ECD es una escala de 16 ítems distribuidos en 5 subescalas: incertidumbre respecto a qué curso de acción tomar, conocimiento acerca de las opciones, valores que la persona le asigna a las opciones, participación de otros en el proceso de toma de decisión y decisión efectiva y satisfactoria para el sujeto que ya ha tomado la decisión (Anexo 1).

Este estudio considera la validación de las cuatro primeras subescalas, considerando que la subescala de decisión efectiva mide la satisfacción con una decisión ya tomada.

Las cuatro subescalas están compuestas por tres ítems cada una y se presentan como escala de Likert de 5 puntos (desde 1 de Total Acuerdo a 5 Total Desacuerdo), en que los puntajes altos indican conflicto decisional y factores contribuyentes.

Traducción y adaptación de la escala. Una de las autoras tradujo la escala y la contra traducción fue 


\section{ANEXO 1 \\ Escala de C onflicto Decisional "M i dificultad en tomar esta decisión"}

Ahora, pensando en la elección que usted acaba de hacer, por favor lea los siguientes comentarios hechos por algunas personas cuando tomaron sus decisiones. Se le solicita que frente a cada uno de ellos, indique cuán de acuerdo 0 desacuerdo se encuentra con la aseveración. Para ello, encierre en un círculo el número de la alternativa que mejor representa lo que usted siente acerca de ese comentario en relación con su decisión (el número 1 indica que usted está totalmente de acuerdo con la aseveración y el número 5, que usted está totalmente en desacuerdo con la aseveración).

\begin{tabular}{|c|c|c|c|c|c|}
\hline & 1 & 2 & 3 & 4 & 5 \\
\hline Esta es una decisión fácil de tomar para mí & $\begin{array}{l}\text { Totalmente } \\
\text { de acuerdo }\end{array}$ & $\begin{array}{c}\text { De } \\
\text { acuerdo }\end{array}$ & Indiferente & $\begin{array}{c}\text { En } \\
\text { desacuerdo }\end{array}$ & $\begin{array}{l}\text { Totalmente } \\
\text { en desacuerdo }\end{array}$ \\
\hline $\begin{array}{l}\text { Yo estoy segura de lo que tengo que } \\
\text { hacer en esta decisión }\end{array}$ & $\begin{array}{l}\text { Totalmente } \\
\text { de acuerdo }\end{array}$ & $\begin{array}{l}\text { De } \\
\text { acuerdo }\end{array}$ & Indiferente & $\begin{array}{c}\text { En } \\
\text { desacuerdo }\end{array}$ & $\begin{array}{l}\text { Totalmente } \\
\text { en desacuerdo }\end{array}$ \\
\hline Está claro cuál es la mejor opción para mí & $\begin{array}{l}\text { Totalmente } \\
\text { de acuerdo }\end{array}$ & $\begin{array}{l}\text { De } \\
\text { acuerdo }\end{array}$ & Indiferente & $\begin{array}{c}\text { En } \\
\text { desacuerdo }\end{array}$ & $\begin{array}{l}\text { Totalmente } \\
\text { en desacuerdo }\end{array}$ \\
\hline $\begin{array}{l}\text { Yo estoy consciente de las diferentes } \\
\text { opciones que tengo en esta decisión }\end{array}$ & $\begin{array}{l}\text { Totalmente } \\
\text { de acuerdo }\end{array}$ & $\begin{array}{l}\text { De } \\
\text { acuerdo }\end{array}$ & Indiferente & $\begin{array}{c}\text { En } \\
\text { desacuerdo }\end{array}$ & $\begin{array}{l}\text { Totalmente } \\
\text { en desacuerdo }\end{array}$ \\
\hline $\begin{array}{l}\text { Yo siento que conozco los aspectos } \\
\text { positivos de cada opción }\end{array}$ & $\begin{array}{l}\text { Totalmente } \\
\text { de acuerdo }\end{array}$ & $\begin{array}{c}\text { De } \\
\text { acuerdo }\end{array}$ & Indiferente & $\begin{array}{c}\text { En } \\
\text { desacuerdo }\end{array}$ & $\begin{array}{l}\text { Totalmente } \\
\text { en desacuerdo }\end{array}$ \\
\hline $\begin{array}{l}\text { Yo siento que conozco los aspectos } \\
\text { negativos de cada opción }\end{array}$ & $\begin{array}{l}\text { Totalmente } \\
\text { de acuerdo }\end{array}$ & $\begin{array}{c}\text { De } \\
\text { acuerdo }\end{array}$ & Indiferente & $\begin{array}{c}\text { En } \\
\text { desacuerdo }\end{array}$ & $\begin{array}{l}\text { Totalmente } \\
\text { en desacuerdo }\end{array}$ \\
\hline $\begin{array}{l}\text { Yo tengo claridad acerca de cuán importantes } \\
\text { son los aspectos positivos para mí }\end{array}$ & $\begin{array}{l}\text { Totalmente } \\
\text { de acuerdo }\end{array}$ & $\begin{array}{l}\text { De } \\
\text { acuerdo }\end{array}$ & Indiferente & $\begin{array}{c}\text { En } \\
\text { desacuerdo }\end{array}$ & $\begin{array}{l}\text { Totalmente } \\
\text { en desacuerdo }\end{array}$ \\
\hline $\begin{array}{l}\text { Yo tengo claridad acerca de cuán importantes } \\
\text { son los aspectos negativos para mí }\end{array}$ & $\begin{array}{l}\text { Totalmente } \\
\text { de acuerdo }\end{array}$ & $\begin{array}{c}\text { De } \\
\text { acuerdo }\end{array}$ & Indiferente & $\begin{array}{c}\text { En } \\
\text { desacuerdo }\end{array}$ & $\begin{array}{l}\text { Totalmente } \\
\text { en desacuerdo }\end{array}$ \\
\hline $\begin{array}{l}\text { Yo tengo claridad acerca de qué es lo más } \\
\text { importante para mí (los aspectos } \\
\text { positivos o los aspectos negativos) }\end{array}$ & $\begin{array}{l}\text { Totalmente } \\
\text { de acuerdo }\end{array}$ & $\begin{array}{c}\text { De } \\
\text { acuerdo }\end{array}$ & Indiferente & $\begin{array}{c}\text { En } \\
\text { desacuerdo }\end{array}$ & $\begin{array}{l}\text { Totalmente } \\
\text { en desacuerdo }\end{array}$ \\
\hline $\begin{array}{l}\text { Yo estoy tomando esta decisión sin ninguna } \\
\text { presión de otras personas }\end{array}$ & $\begin{array}{l}\text { Totalmente } \\
\text { de acuerdo }\end{array}$ & $\begin{array}{c}\text { De } \\
\text { acuerdo }\end{array}$ & Indiferente & $\begin{array}{c}\text { En } \\
\text { desacuerdo }\end{array}$ & $\begin{array}{l}\text { Totalmente } \\
\text { en desacuerdo }\end{array}$ \\
\hline $\begin{array}{l}\text { Yo tengo la cantidad apropiada de apoyo de otros } \\
\text { para tomar esta decisión }\end{array}$ & $\begin{array}{l}\text { Totalmente } \\
\text { de acuerdo }\end{array}$ & $\begin{array}{c}\text { De } \\
\text { acuerdo }\end{array}$ & Indiferente & $\begin{array}{c}\text { En } \\
\text { desacuerdo }\end{array}$ & $\begin{array}{l}\text { Totalmente } \\
\text { en desacuerdo }\end{array}$ \\
\hline $\begin{array}{l}\text { Yo cuento con suficientes consejos acerca } \\
\text { de las opciones }\end{array}$ & $\begin{array}{l}\text { Totalmente } \\
\text { de acuerdo }\end{array}$ & $\begin{array}{c}\text { De } \\
\text { acuerdo }\end{array}$ & Indiferente & $\begin{array}{c}\text { En } \\
\text { desacuerdo }\end{array}$ & $\begin{array}{l}\text { Totalmente } \\
\text { en desacuerdo }\end{array}$ \\
\hline Yo siento que he hecho una elección bien informada & $\begin{array}{l}\text { Totalmente } \\
\text { de acuerdo }\end{array}$ & $\begin{array}{c}\text { De } \\
\text { acuerdo }\end{array}$ & Indiferente & $\begin{array}{c}\text { En } \\
\text { desacuerdo }\end{array}$ & $\begin{array}{l}\text { Totalmente } \\
\text { en desacuerdo }\end{array}$ \\
\hline Mi decisión muestra lo que es importante para mí & $\begin{array}{l}\text { Totalmente } \\
\text { de acuerdo }\end{array}$ & $\begin{array}{c}\text { De } \\
\text { acuerdo }\end{array}$ & Indiferente & $\begin{array}{c}\text { En } \\
\text { desacuerdo }\end{array}$ & $\begin{array}{l}\text { Totalmente } \\
\text { en desacuerdo }\end{array}$ \\
\hline Yo espero mantenerme firme en mi decisión & $\begin{array}{l}\text { Totalmente } \\
\text { de acuerdo }\end{array}$ & $\begin{array}{c}\text { De } \\
\text { acuerdo }\end{array}$ & Indiferente & $\begin{array}{c}\text { En } \\
\text { desacuerdo }\end{array}$ & $\begin{array}{l}\text { Totalmente } \\
\text { en desacuerdo }\end{array}$ \\
\hline Yo estoy satisfecha con mi decisión & $\begin{array}{l}\text { Totalmente } \\
\text { de acuerdo }\end{array}$ & $\begin{array}{c}\text { De } \\
\text { acuerdo }\end{array}$ & Indiferente & $\begin{array}{c}\text { En } \\
\text { desacuerdo }\end{array}$ & $\begin{array}{l}\text { Totalmente } \\
\text { en desacuerdo }\end{array}$ \\
\hline
\end{tabular}

Copyright A. O'Connor, 1993; Revisada en Marzo 1997; Versión 1998

Traducción Mila Umutia, 1999. 
realizada por una enfermera bilingüe, que había sido capacitada en el tema de toma de decisiones y conflicto decisional por la autora del instrumento original. Se adaptó el instrumento traducido y se probó en el grupo de alumnos del año 2000, lo que demostró una buena comprensión de los ítems.

Contexto. Para su validación se obtuvo una muestra que consta de 331 alumnos de la Pontificia Universidad Católica de Chile, que ingresaron al primer año de la carrera de Enfermería, en los meses de marzo entre los años 2000 y 2004. La metodología para recolectar la información fue la misma todos los años. Una de las investigadoras se reunió con el curso de $1^{\text {er }}$ año en la primera clase del semestre y les informó verbalmente que este estudio tenía por objeto identificar conflicto decisional en el alumnado respecto a mantenerse estudiando la carrera de enfermería versus cambiarse de carrera, dar la prueba nacional de selección universitaria nuevamente u otras opciones. Esta información permitiría diseñar estrategias de apoyo decisional para ayudar a aquellos alumnos que se encontraran con dificultades para tomar esta decisión. Previo a que respondieran la escala, se les solicitó verbalmente su consentimiento informado, dejando en libertad a aquellos que no quisieran responder.

La distribución de los sujetos de la muestra (n =331) según año de ingreso fue: el año 2000 participaron 48 alumnos (38\% del ingreso); el año 2001, 42 (36,5\% del ingreso); el año 2002, 71 (56,3\% del ingreso); el año 2003, 57 (55,3\% del ingreso) y el año 2004 participaron 113 (91\% del ingreso).

\section{Resultados}

De los 588 alumnos ingresados a través de los años 2000 a 2004 contestaron el cuestionario 331. De ellos, se excluyeron aquellos que presentaron respuestas incompletas en al menos uno de los 12 ítems, lo que correspondió a 3\% de los sujetos, por lo que la muestra se redujo a 321 casos válidos.
Con la Prueba de Normalidad de KolmogorovSmirnov se pudo concluir que ninguna de las variables en estudio presentó una distribución normal. No obstante, la distribución obtenida por el puntaje total de la prueba fue normal.

\section{Confiabilidad:}

Para determinar la confiabilidad -como coherencia interna- se utilizó el método Alfa de Cronbach*, cuyo índice para el total de 321 casos, alcanzó a 0,8076 , indicando una alta confiabilidad interna de la escala. Según la Tabla 1, sólo la eliminación del ítem presión (ítem $\mathrm{n}^{\mathrm{0}} 10$ ) incrementaría levemente el valor de la confiabilidad interna $(0,8088)$.

$\mathrm{Al}$ calcular el coeficiente Alfa de Cronbach para cada subescala planteada teóricamente en la adaptación del test, se obtuvieron valores que oscilaron entre 0,51 y 0,77 , siendo la subescala participación de otros, la que mostró la menor coherencia interna (Tabla 2).

$\mathrm{Al}$ calcular las correlaciones entre las subescalas de la ECD, todas los valores fueron positivos y significativos $(p<0,01)$, indicando que a mayor conocimiento de las opciones disponibles, claridad en los valores asociados a dichas opciones y una participación de otros positiva, mayor certidumbre ante la decisión (Tabla 3).

El valor más bajo lo obtuvo la subescala participación de otros, con un índice de 0,51 y el más alto correspondió a la subescala conocimientos, con un índice de 0,77.

\section{Validez}

Análisis factorial. Al analizar la aplicabilidad del análisis factorial a los datos, se observaron los estadísticos KMO, cuyo valor fue de 0,765 y el contraste de Barlett (que indica la no-significancia de la hipótesis nula de variables incorrelacionadas), ambos indican la adecuación de los datos para realizar un análisis factorial (Tabla 4).

Para determinar el número de factores a extraer se consideró el Criterio de Kaiser, el cual establece que el número de factores a extraer corresponde al número de autovalores mayores que 1, debiendo extraerse 4 factores comunes. Este número de factores, sería coherente con la forma de concebir la ECD.

*A través del programa computacional SPSS para Windows, versión 11.5. 
Tabla 1. Valor Alfa C ronbach al eliminar un Ítem

\begin{tabular}{|c|c|c|c|c|c|}
\hline Código & ITEM & Promedio & Varianza & $\begin{array}{l}\text { Correlación } \\
\text { Item-total }\end{array}$ & $\begin{array}{l}\text { Alpha } \\
\text { Cronbach }\end{array}$ \\
\hline Consc & $\begin{array}{l}\text { Yo estoy consciente de las diferentes } \\
\text { opciones que tengo en esta decisión }\end{array}$ & 22,1807 & 34,4985 &, 5055 & ,7897 \\
\hline Conozpo & $\begin{array}{l}\text { Yo siento que conozco los aspectos positivos } \\
\text { de cada opción }\end{array}$ & 21,9875 & 34,5311 & ,4908 & ,7908 \\
\hline Conoczne & $\begin{array}{l}\text { Yo siento que conozco los aspectos negativos } \\
\text { de cada opción }\end{array}$ & 21,8037 & 34,2520 & 4787 & ,7915 \\
\hline Claripo & $\begin{array}{l}\text { Yo tengo claridad acerca de cuán importantes } \\
\text { son los aspectos positivos para mí }\end{array}$ & 22,2118 & 34,3300 &, 5433 & ,7869 \\
\hline Clarine & $\begin{array}{l}\text { Yo tengo claridad acerca de cuán importantes } \\
\text { son los aspectos negativos para mí }\end{array}$ & 21,9875 & 34,5436 & ,4787 & ,7917 \\
\hline Masimp & $\begin{array}{l}\text { Yo tengo claridad acerca de qué es lo más } \\
\text { importante para mí (los aspectos positivos } \\
\text { o los aspectos negativos) }\end{array}$ & 22,1776 & 34,9027 & ,4543 & ,7938 \\
\hline Presión & $\begin{array}{l}\text { Yo estoy tomando esta decisión sin ninguna } \\
\text { presión de otras personas }\end{array}$ & 22,4704 & 36,3999 & ,2669 & ,8098 \\
\hline Apoyo & $\begin{array}{l}\text { Yo tengo la cantidad apropiada de apoyo de } \\
\text { otros para tomar esta decisión }\end{array}$ & 22,4860 & 36,2381 & 3210 & 8045 \\
\hline Consejo & $\begin{array}{l}\text { Yo cuento con suficientes consejos acerca } \\
\text { de las opciones }\end{array}$ & 21,9252 & 34,3131 & ,4549 & ,7936 \\
\hline Decifaci & Esta es una decisión fácil de tomar para mí & 20,9065 & 32,9037 & ,4186 & 8000 \\
\hline Segura & $\begin{array}{l}\text { Yo estoy segura de lo que tengo que hacer } \\
\text { en esta decisión }\end{array}$ & 21,8131 & 32,6775 & ,5562 & 7836 \\
\hline Claro & Está claro cuál es la mejor opción para mí & 21,7414 & 32,3611 & ,5299 & ,7862 \\
\hline
\end{tabular}

Tabla 2. Valores Alfa de Cronbach, escala total y subescalas

\begin{tabular}{|ccccc|}
\hline Escala total & \multicolumn{4}{c|}{ Subescalas } \\
\hline & Incertidumbre & Conocimientos & Valores & Participación de otros \\
0,8076 &, 7425 &, 7710 &, 7652 &, 5048 \\
\hline
\end{tabular}

Tabla 3. C orrelaciones entre subescalas

\begin{tabular}{|c|c|c|c|c|}
\hline & Incertidumbre & Conocimientos & Valores & Participación de otros \\
\hline Incertidumbre & 1 & ,324** & ,336** &, $401^{\text {** }}$ \\
\hline Conocimientos & & 1 & $.460^{\text {** }}$ & ,286** \\
\hline Valores & & & 1 & 291 ** \\
\hline Participación de otros & & & & 1 \\
\hline
\end{tabular}

**Correlación es significativa al nivel 0,01 (2-colas) 
Tabla 4. Test KMO y de Bartlett

$\begin{array}{llr}\text { Kaiser-Meyer-Olkin Measure of Sampling Adequacy } & 765 \\ \text { Bartlett's Test of Sphericity } & \text { Approx. Chi-Square } & 1216,163 \\ & \text { df } & 66 \\ & \text { Sig. } & , 000\end{array}$

Tabla 5. Total de la Varianza Explicadaa

\begin{tabular}{|c|c|c|c|c|c|c|c|}
\hline \multirow[t]{2}{*}{ Factor } & \multicolumn{3}{|c|}{ Autovalores iniciales } & \multicolumn{3}{|c|}{$\begin{array}{c}\text { Suma de Cuadrados de las Carga- } \\
\text { Extracción }\end{array}$} & \multirow{2}{*}{$\begin{array}{c}\text { SSCC }- \text { SL. } \\
\text { Rotation }^{\text {b }} \\
\text { Total }\end{array}$} \\
\hline & Total & $\begin{array}{l}\% \text { de } \\
\text { varianza }\end{array}$ & $\begin{array}{c}\% \\
\text { Acumulativo }\end{array}$ & Total & $\begin{array}{c}\% \text { de } \\
\text { varianza }\end{array}$ & $\begin{array}{c}\% \\
\text { Acumulativo }\end{array}$ & \\
\hline 1 & 3,999 & 33,329 & 33,329 & 3,559 & 29,656 & 29,656 & 2,604 \\
\hline 2 & 1,697 & 14,143 & 47,472 & 1,275 & 10,623 & 40,279 & 2,475 \\
\hline 3 & 1,144 & 9,533 & 57,005 & ,756 & 6,297 & 46,575 & 2,663 \\
\hline 4 & 1,065 & 8,879 & 65,884 & ,648 & 5,401 & 51,977 & 1,540 \\
\hline 5 & ,821 & 6,845 & 72,728 & & & & \\
\hline 6 & ,673 & 5,607 & 78,335 & & & & \\
\hline 7 & 616 & 5,134 & 83,470 & & & & \\
\hline 8 &, 527 & 4,396 & 87,865 & & & & \\
\hline 9 &, 503 & 4,191 & 92,057 & & & & \\
\hline 10 & ,407 & 3,391 & 95,448 & & & & \\
\hline 11 & ,346 & 2,879 & 98,327 & & & & \\
\hline 12 & ,201 & 1,673 & 100,000 & & & & \\
\hline
\end{tabular}

aMétodo de extracción: Factorización de ejes principales.

${ }^{b}$ Cuando los factores están correlacionados, no se pueden sumar las sumas de los cuadrados de las saturaciones para obtener una varianza total explicada

Varianza total explicada. El conjunto de los cuatro factores da cuenta de $52 \%$ del total de la varianza. Destacan los primeros dos factores, dado que en conjunto explican $40 \%$ del total de la varianza obtenida (Tabla 5).

Matriz de configuración rotada. De acuerdo con las cargas factoriales de la matriz de configuración rotada (método Promax) se obtienen 4 factores que darían cuenta de la variabilidad de la escala ECD (Tabla 6). A saber: El primer factor: conocimiento de las opciones, incluye 3 items que evalúan conocimiento de las opciones, que cargan en forma importante en los factores 1 y 2 . Los siguientes tres factores: incertidumbre ante la decisión a tomar, importancia personal de las opciones y participación de otros ante la decisión, están compuestos cada uno de ellos por 3 ítems. Se destaca que en el factor participación de otros, el ítem consejo, presenta cargas similares en los cuatro factores.

La matriz de correlaciones entre los factores, indica que, en general, los factores están moderadamente correlacionados entre sí. Las correlaciones entre los factores 1 (conocimiento de las opciones) y 4 (participación de otros); y los factores 3 (valores personales) y 4 (participación de otros) son bajas (Tabla 7).

Presencia de Conflicto Decisional (CD). La presencia de $C D$ se obtuvo a partir del análisis de la subescala Incertidumbre acerca de cuál curso de acción tomar frente a la decisión, que consta de tres ítems. 
Tabla 6. M atriz de configuración ${ }^{a, b}$

\begin{tabular}{|c|c|c|c|c|c|}
\hline \multirow[t]{2}{*}{ Items } & & \multicolumn{4}{|c|}{ Factor } \\
\hline & & 1 & 2 & 3 & 4 \\
\hline 5. & Conozpo & ,961 & & & \\
\hline 6. & Conoczne & ,777 & & 102 & \\
\hline 4. & Consc & ,418 & 306 & & \\
\hline 1. & Decifaci & & 741 & & \\
\hline 3. & Claro & & ,705 & & \\
\hline 2. & Segura & & 669 & & \\
\hline 7. & Claripo & & & 873 & \\
\hline 8. & Clarine & ,136 & & 703 & \\
\hline 9. & Masimp & -,154 & 186 & 610 & \\
\hline 11. & Ароуо & & & & 900 \\
\hline 10. & Presión & & 192 & & 301 \\
\hline 12. & Consejo & 135 & 203 & 123 & ,234 \\
\hline
\end{tabular}

àétodo de extracción: Factorización de ejes principales. Método de rotación: Promax con normalización Kaiser.

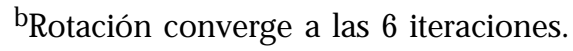

Tabla 7. M atriz de correlaciones de los factoresa

\begin{tabular}{|cccccc|}
\hline Factor & $\mathbf{1}$ & $\mathbf{2}$ & $\mathbf{3}$ & $\mathbf{4}$ \\
\hline & 1 & 1 &, 334 &, 548 &, 249 \\
& 2 & 1 &, 424 &, 427 \\
3 & & & 1 &, 267 \\
4 & & & & 1 \\
\hline
\end{tabular}

aMétodo de extracción: Factorización de ejes principales. Método de rotación: Promax con normalización Kaiser.

La presencia de CD por año de ingreso fue: año 2000 45,8\%; año 2001 65\%; año 2002 66,7\%; año 2003 60\% y año 2004 60,6\%. En promedio, considerando todos los años, $60 \%$ de los estudiantes presenta conflicto en la decisión de estudiar la carrera de enfermería.

\section{DisCUSIÓN}

Los resultados de la prueba Alfa de Cronbach $(0,80)$ son similares a los encontrados por $\mathrm{O}^{\prime}$ Connor en la validación de la escala original ${ }^{5}$, los que iban desde 0,78 a 0,92, considerando los distintos grupos a los que se aplicó la prueba.

Estos resultados además apoyan la relación planteada teóricamente entre los factores definidos como contribuyentes al conflicto decisional y la presencia de éste. Es así como un mayor conocimiento de las opciones disponibles, una mayor claridad en los valores asociados a dichas opciones y una participación de otros positiva, se asocian a una menor incertidumbre ante la decisión.

Con la factorización de ejes principales se identifican con el criterio de Kaiser 4 factores, que 
dan cuenta de 52\% del total de la varianza. Al rotar la matriz con método Promax se obtienen también cuatro factores. El primero está constituido por los ítems correspondientes a la subescala conocimientos, sin embargo el ítem "yo estoy consciente de las diferentes opciones que tengo" carga también en el segundo factor. Esto podría explicarse porque no tener conciencia de las diferentes opciones puede considerarse en sí como parte del conflicto y a la vez como un factor que contribuye a él. Se sugiere analizar el comportamiento de este ítem en otros estudios.

Estos resultados son consistentes con el marco conceptual propuesto por O'Connor, a excepción del ítem "consejo" que al cargar en los cuatro factores no se identifica claramente en el factor cuatro, el que corresponde a la escala participación de otros. Este hallazgo puede obedecer a la forma en que está redactado el ítem y al uso del término consejo, el que podría haber sido interpretado de diferentes maneras. El consejo puede interpretarse como el aclarar opciones, proponer opciones, brindar apoyo e incluso ejercer presión hacia una determinada opción. Además el consejo podría interpretarse como entregado por pares, familiares o profesionales.

A partir de estos resultados se propone eliminar o precisar la redacción del ítem consejo para futuras aplicaciones, ya que la subescala participación de otros se refiere a la percepción de sentirse presionado o apoyado, y ambas situaciones pueden ser entregadas a través de un consejo.

La ECD está planteada como una escala genérica para identificar conflicto decisional, este estudio aportó a determinar su confiabilidad y validez en un grupo específico y frente a una decisión determinada.

Los resultados obtenidos en este estudio respecto a la presencia de CD frente a la decisión de mantenerse en la carrera de enfermería (en promedio 60\%), son coherentes con las preferencias referidas por los estudiantes al ingreso a la carrera, ya que aproximadamente $50 \%$ de ellos señala no mencionar enfermería como primera prioridad $^{1-3}$. Esta situación también se observó en el estudio de Jara, Polanco y Alveal, quienes encontraron que sólo $40 \%$ de los estudiantes que estaban ingresando a la carrera de enfermería señalaba haberla seleccionado en primer lugar, y sólo $45 \%$ de los estudiantes que estaban en tercer año y $51,4 \%$ del $5^{0}$ nivel señaló haber elegido enfermería como primera opción al momento de postular a la universidad ${ }^{18}$. Por su parte Rongstad refiere, en un estudio realizado en Noruega, que los estudiantes aceptaban ingresar a enfermería a pesar de preferir otras materias y que $45 \%$ de la muestra deseaba estudiar otras carreras ${ }^{19}$. Esta situación ya fue observada por Verderese y García, quienes en un estudio realizado el año 1971 en 13 países de América Latina, en un total de 58 escuelas de enfermería, observaron que $60 \%$ de los estudiantes pensó en seguir otra profesión antes de decidirse por enfermería. El mismo estudio mostró además que aun después de decidir definitivamente estudiar enfermería, 37\% de los estudiantes dudó que esa decisión fuera la más conveniente ${ }^{20}$.

Los jóvenes buscan carreras que les ofrezcan oportunidades de progresión, buenos sueldos, estatus y que no sean interferentes con su vida personal $^{21-23}$ y perciben que enfermería no responde a estas expectativas. Por otra parte, la deserción de la carrera de enfermería se ha relacionado principalmente con motivos vocacionales ${ }^{24,25}$.

Lo anterior señala la importancia de implementar buenos programas de difusión de la carrera, diseñados de acuerdo a las características de la generación que se quiere atraer. A la vez que identificar tempranamente los factores asociados al conflicto decisional que presentan algunos alumnos de mantenerse en la carrera una vez ingresados, con el fin de ofrecerles apoyo en el proceso de decidir la mejor opción (mantenerse 0 no en la carrera) para ellos. 


\section{REFERENCIAS}

1. FERNÁNDEZ P. Reporte proceso de admisión ordinaria carrera de enfermería. Pontificia Universidad Católica de Chile, Santiago 2002.

2. FERNÁNDEZ P. Reporte proceso de admisión ordinaria carrera de enfermería. Pontificia Universidad Católica de Chile, Santiago 2003.

3. FERNÁNDEZ P. Reporte proceso de admisión ordinaria carrera de enfermería. Pontificia Universidad Católica de Chile, Santiago 2004.

4. JANIS I, MANN L. Decision Making: a psychological analysis of conflict, choice, and commitment. Free Press. NY. 1977.

5. O'Connor A. Validation of a Decisional Conflict Scale. Medical Decision Making 1995; 15: 25-30.

6. O'Connors AM. Elecciones y decisiones en salud: Una alianza entre profesionales y usuarios aplicando transferencia tecnológica canadiense. Documento Interno. Escuela de Enfermería. Pontificia Universidad Católica de Chile. 2001.

7. Koedoot N, Molenaar S, Oosterveld P, Bakker P, De Graefr A, Nooy M et al. The decisional conflict scale: further validation in two samples of Dutch oncology patients. Patient Education and Counseling 2001; 45: 187-93.

8. O'Connor AM, Tugwell P, Wells GA, Elmsie T, HownGWORTH G, MCPHERSON R ET AL. A decision aids for women considering hormone therapy after menopause: decision support framework and evaluation. Patient Education and Counseling 1998; 33: 267-79.

9. Murray E, Davis H, Tai SS, Coulter A, Gray a, Haines A. Randomized controlled trial of an interactive multimedia decision aid on hormone replacement therapy in primary care BMJ 2001; 323: 490-3.

10. Song MK, SEREIKA SM. An evaluation of the Decisional Conflict Scale for measuring the quality of endof-life decision making. Patient Education and Counseling 2006; 61: 397-404.

11. Peddie V, Van Tejulngen E, Bhattacharya S. Ending invitro fertilization: Women's perception's of decision making. Human Fertility 2004; 7: 31-7.

12. Campos S, Pérez J. Autoeficacia y conflicto decisional frente a la disminución de peso corporal en mujeres. Rev Chil Nutr 2007; 34: 213-8.
13. Grant FC, Laupacis A, O'Connor A, Rubens F, Robbiee $\mathrm{J}$. Evaluation of a decision aid for patients considering autologous blood donation before open.-heart surgery. CMAJ 2001; 164: 1139-44.

14. Montori VM, Bryant SC, O'Connor A, Jorgensen N, Walsh E, Smith S. Decisional Attributes of Patients with Diabetes Diabetes Care 2003; 26: 2804-9.

15. Campos C, Urrutia M, Lange I, Campos S, Campos MS. Trabajo Académico en Red en torno a la Toma de Decisiones en Salud. Ciencia y Enfermería 2005; 11 (1): 25-35.

16. Mendoza PS, Jofré AV, Valenzueia SS. La toma de decisiones en salud y el modelo conceptual de Ottawa. Investigación y Educación en Enfermería 2006; 24: 86-92.

17. Jofré V, Mendoza S. Toma de Decisiones en Salud en Mujeres Cuidadoras Informales. Ciencia y Enfermería 2005 ; 11: 37-49.

18. Jara P, Polanco O, Alveal M. Pencepción del rol profesional de alumnos de enfermería de la Universidad de Concepción, Chile. Investigación y Educación en Enfermería 2005; 23: 56-69.

19. RoNGSTAD MK. Recruitment to and Motivation for Nursing Education and the Nursing Profession J Nurs Educ 2002; 41: 321-5.

20. Verderese $O$, García J. La decisión de estudiar enfermería. Educación Médica y Salud 1974; 8: 390-407.

21. Hemsley-Brown J, FosketT N. Career desirability: young people's perceptions of nursing as a career. J Adv Nurs 1999; 29: 1342-50.

22. Slader J. Who Wants to be a Nurse: Motivation of the New Generation. J Prof Nurs 2003; 19: 173-5.

23. Cabieses B, Muñoz M, González P, Gómez C. Motivaciones y Medios de Comunicación preferidos para conocer la Carrera de Enfermería en la Pontificia Universidad Católica de Chile (UC) Rev Cognición № 9. Disponible en: http://216.75.15.111/ cognicion/ index.php?option $=$ com content\&task =view \&id=28\&ltemid=42. (Consultado el 2 de junio de 2008).

24. SanabRia H. Deserción de estudiantes de enfermería en cuatro universidades de Perú. Anales de la Facultad de Medicina. Universidad Nacional Mayor de San Marcos 2002; 63: 301-11.

25. Vanhanen L, Janhonen S. Factors associated with student's orientations to nursing. J Adv Nurs 2000; 31: 1054-62. 